\title{
SOI MICROMACHINED 5-AXIS MOTION SENSOR USING RESONANT ELECTROSTATIC DRIVE AND NON-RESONANT CAPACITIVE DETECTION MODE
}

\author{
Yoshiyuki Watanabe ${ }^{1}$, Toshiaki Mitsui ${ }^{1}$, Takashi Mineta ${ }^{2}$, Yoshiyuki Matsu ${ }^{3}$ and Kazuhiro Okada ${ }^{3}$ \\ ${ }^{1}$ Yamagata Research Institute of Technology, MEMS Laboratory, 2-2-1 Matsuei, Yamagata 990-2473, Japan \\ phone: +81-23-647-3125, fax: +81-23-647-3129, e-mail: wat@mmp.yamagata-rit.go.jp \\ ${ }^{2}$ Faculty of Science and Technology,Hirosaki University, 3 Bunkyo-cho, Hirosaki, Aomori 036-8561, Japan \\ ${ }^{3}$ Wacoh corporation, Tsuzuki Bldg.4F, 4-244-1 Sakuragi-cho, Saitama 331-0852, Japan
}

\begin{abstract}
This paper presents a 5 -axis motion sensor which can detect 3-axis acceleration and 2-axis angular rate capacitively. This sensor is fabricated by SOI bulk-micromachining, and is vacuum sealed by anodic bonding and activation of the non-evaporated getters. By applying Z-axis reference vibration of a proof mass with an electrostatic force at resonant frequency, 5-axis motions can be detected capacitively at non-resonant detection mode. Measured sensitivity of $\mathrm{X}^{-}, \mathrm{Y}^{-}$and $\mathrm{Z}$-axis acceleration were approximately $1.9,1.1$ and 4.7 $\mathrm{V} / \mathrm{G}$, respectively. $\mathrm{X}^{-}$and $\mathrm{Y}$-axis angular rate sensitivity were approximately 6.7 and $12 \mathrm{mV} /(\mathrm{deg} / \mathrm{s})$. The sensor chip size is $5.0 \times 5.0 \times 1.7 \mathrm{~mm}^{3}$.
\end{abstract}

Keywords: 5-axis motion, 3-axis acceleration, 2-axis angular rate, resonant drive, non-resonant detection

\section{INTRODUCTION}

MEMS type accelerometers and angular rate sensors (gyroscopes) have come into use in wide fields, such as automobile chassis control, auxiliary function of GPS (Global Positioning System) navigation, motion control of the robot and $\mathrm{I} / \mathrm{O}$ devices for amusement field. In quite recent years, multi-axis motion sensing have been required in many wide fields. Multi-axis motion sensing can be performed, of course, using multiple uni-axis sensors. However, its multi-axis sensing module has disadvantages such as large size and high production cost. Therefore the multi-axis motion sensor which can detect multi-axis acceleration and multi-axis angular rate with only one chip, has serviceable advantages. We have already reported a 5 -axis motion sensor with $\mathrm{Si}-\mathrm{Si}$ direct bonded structure using silicon bulk-micromachining, and we have demonstrated the principles of detection of $3-$ axis acceleration and 2 -axis angular rate $[1,2]$. This sensor have some disadvantages for practical using. First one was difficult to seal the sensor chip in vacuum because of its open structure, and the second was difficult to fabricate the sensor with high accuracy. In order to improve these problems, we have developed a new 5-axis motion sensor with SOI structure fabricated by SOI bulk-micromachining using deep RIE and vacuum anodic bonding processes.

\section{SENSOR STRUCTURE}

The sensor structure is shown in Fig.1. The sensor consists of three layers which are a glass, an SOI (Silicon On Insulator) and a glass. The inner cavity is vacuum sealed by anodic bonding and activation of the non-evaporated getters in order to prevent the squeezed film damping effect. A silicon proof mass, located in the center of the sensor, is suspended by a cross shape silicon beam which is formed in an active silicon layer of the SOI wafer. Each glass layer has five electrodes on the surface of inside. The silicon pillars around the mass, are fixed on Glass-2 for interconnection of the electrodes on both glass wafers to the electrodes through the glass holes. The cross shape center electrodes (T0 and B0) and surrounding electrodes (T1-T4 and $\mathrm{B} 1-\mathrm{B} 4)$, are used for the electrostatic driving and the capacitive detection, respectively.

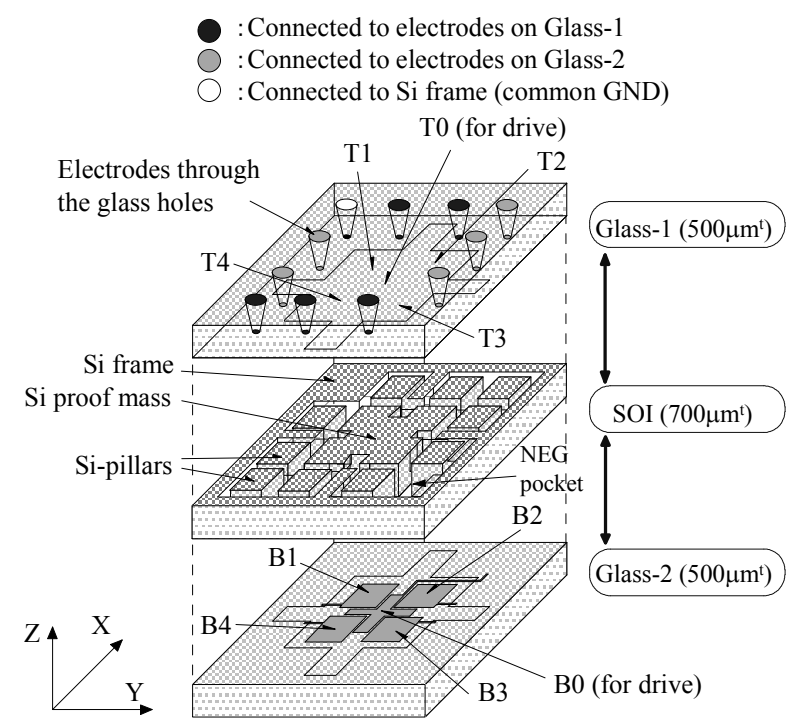

Fig.1 Sensor structure

\section{PRINCIPLES}

The mass movement by 5 -axis motion is shown in Fig.2(a). The proof mass suspended by a cross shape silicon beam, is vibrated constantly along Z-axis by an electrostatic force. $Z$-axis acceleration shifts the mass along $\mathrm{Z}^{-}$axis, and $\mathrm{X}^{-}$and $\mathrm{Y}^{-}$axis acceleration tilts the 
mass. When angular rate around $\mathrm{X}^{-}$and $\mathrm{Y}^{-}$axis is applied, Coriolis' force makes tilting vibration synchronizing with $\mathrm{Z}$-axis reference vibration, along $\mathrm{Y}$-and $\mathrm{X}$-axis, respectively.

Block diagram of detection of 5-axis motions is shown in Fig.2(b). The proof mass is driven electrostatically with two center electrodes of $\mathrm{T} 0$ and $\mathrm{B} 0$ of which phases

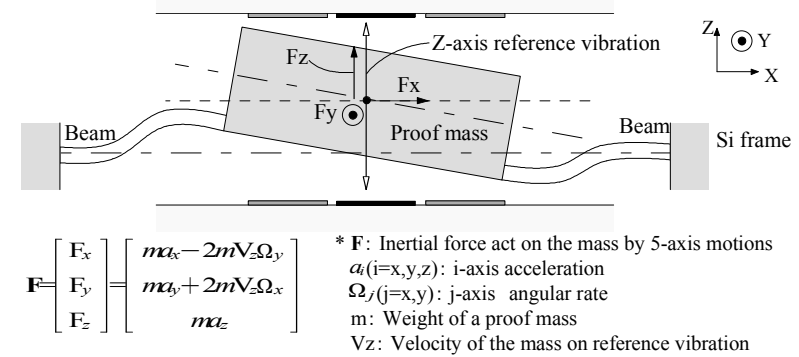

(a) Mass movement by 5 -axis motions

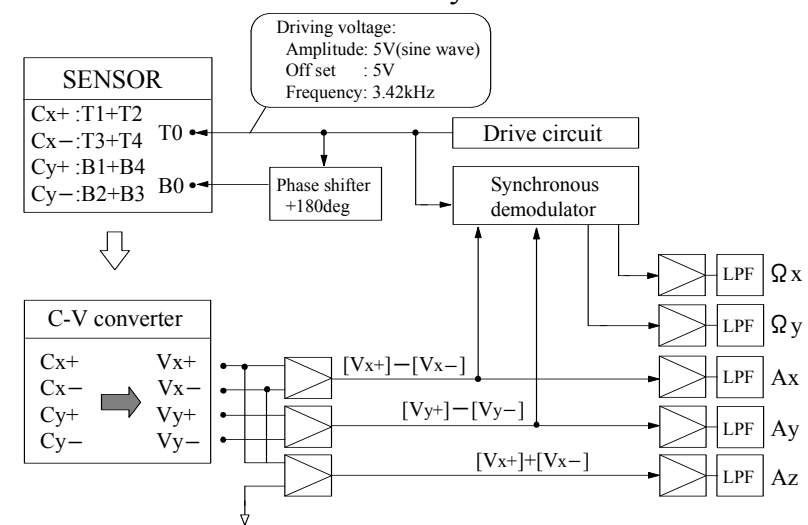

(b) Block diagram of detection

Fig.2 Principle of 5-axis motion sensing

are shifted 180deg each other. Four capacitances of $\mathrm{Cx}+$ $(: \mathrm{T} 1+\mathrm{T} 2), \mathrm{Cx}^{-}(: \mathrm{T} 3+\mathrm{T} 4), \mathrm{Cy}+(: \mathrm{B} 1+\mathrm{B} 4)$ and $\mathrm{Cy}^{-}$ $(: \mathrm{B} 2+\mathrm{B} 3)$ are converted to $\mathrm{Vx}^{+}, \mathrm{Vx}^{-}, \mathrm{Vy}+$ and $\mathrm{Vy}^{-}$, respectively, with a $\mathrm{C}-\mathrm{V}$ converter. Through an amplifier and a low pass filter (LPF), 3-axis acceleration of $\mathrm{Ax}$, Ay and $\mathrm{Az}$ can be detected by $\left[\left(\mathrm{V}_{\mathrm{x}+}\right)-\left(\mathrm{V}_{\mathrm{x}^{-}}\right)\right]$, $\left[\left(\mathrm{V}_{\mathrm{y}^{+}}\right)-\left(\mathrm{V}_{\mathrm{y}^{-}}\right)\right]$and $\left[\left(\mathrm{V}_{\mathrm{x}}+\right)+\left(\mathrm{V}_{\mathrm{x}^{-}}\right)\right]$, respectively. The 2-axis angular rates of $\Omega \mathrm{x}$ and $\Omega y$ can be detected by a synchronous demodulation, an amplification and a low pass filtration of $\left[\left(\mathrm{Vy}^{+}\right)-\left(\mathrm{Vy}^{-}\right)\right]$and $\left[\left(\mathrm{Vx}^{+}\right)-\left(\mathrm{Vx}^{-}\right)\right]$, respectively. Since the angular rate signals are synchronizing with $\mathrm{Z}$-axis driving frequency of $3.42 \mathrm{kHz}$, the angular rate can be separated from low frequency accelerations below several ten $\mathrm{Hz}$ with the synchronous demodulator.

\section{FABRICATION}

This sensor is fabricated by bulk-micromachining of a SOI wafer which consists of a silicon substrate (sub-Si, $\left.675 \mu \mathrm{m}^{\mathrm{t}}\right)$, a buried oxide (BOX, $\left.2 \mu \mathrm{m}^{\mathrm{t}}\right)$ and an active silicon (act-Si, 20 $\mu^{t}$ ) layers. The glass wafers for low temperature anodic bonding (Asahi Technoglass Corp., SW-Z , $\left.500 \mu \mathrm{m}^{\mathrm{t}}\right)$ are used. Fabrication process sequence of the motion sensor is shown in Fig.3.

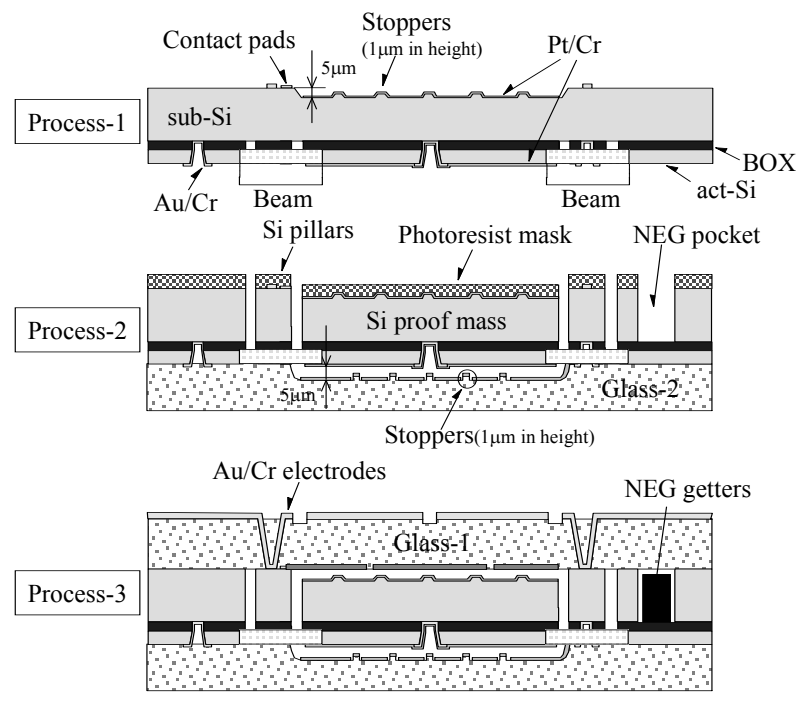

Fig.3 Fabrication process sequence of the sensor

[process-1] The SOI wafer is etched from both sides with 20wt.\%TMAH aq. in order to form the masses, beams, silicon pillars, and the top side gap. The sub-Si and the act-Si layers are electrically interconnected via the BOX layer with $\mathrm{Au} / \mathrm{Cr}$ contact pads. The $\mathrm{Pt} / \mathrm{Cr}$ metal layer on the top side of the mass is formed for protection from sticking during anodic bonding. The $\mathrm{Pt} / \mathrm{Cr}$ metal layer on the bottom side is formed for protection from ion attack after Deep RIE.

[process-2] After anodic bonding of the SOI wafer and the lower glass wafer (Glass-2), sub-Si is etched through by Deep RIE, consequently the proof masses, silicon pillars and the NEG pockets for insetting getters are formed.

[process-3] The NEG getters (Non evaporated getters, SAES Getters Japan) are inset in the NEG pockets. An upper glass wafer (Glass-1) is anodically bonded to an SOI wafer at $300^{\circ} \mathrm{C}, 30 \mathrm{mT}$ Torr with applying voltage of $-1 \mathrm{kV}$ to Glass-1 for vacuum sealing. Finally, after shallow dicing of top side of Glass -1 in order to isolate each electrode, the wafers were separated to each chip.

The act-Si and the sub-Si layers were interconnected with $\mathrm{Au} / \mathrm{Cr}$ contact pads as showon in Fig.4 (a). The configuration after deep etched silicon is shown in Fig.4 (b). The etched profile was extremely perpendicular. The vacuum sealed configuration is shown in Fig.4 (c). The SOI and the Glass-1 wafers were bonded uniformly in entire $\phi 2$ inch wafers. After an activation of NEG getters at $450{ }^{\circ} \mathrm{C}$ in an inert gas, the wafers were separated to sensor chips of $5.0 \times 5.0 \times 1.7 \mathrm{~mm}^{3}$ in size (Fig.4 (d)). 

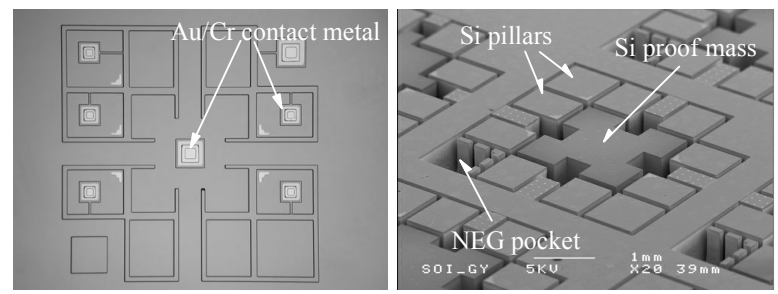

(a) Interconnection of wafers (b) Si deep RIE configuration
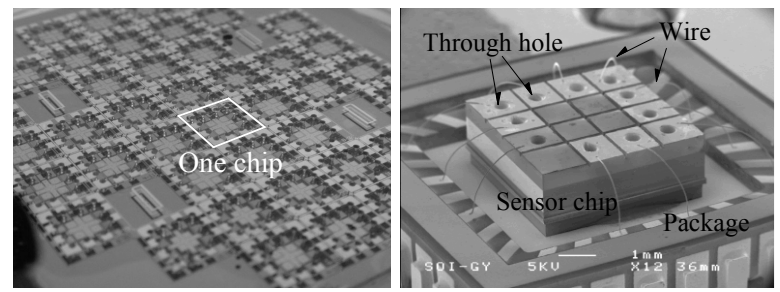

(c)Vacuum sealing

(d)Developed motion sensor

Fig.4 Photographs of the 5-axis motion sensor

\section{EXPERIMENTAL RESULTS}

\section{Vibration characteristics of the resonator}

This sensor is designed for resonant drive and non-resonant detection for suppression of the mechanical coupling $[3,4]$. The large amplitude of reference vibration is required for high sensitive detection of the angular rate. The vibration characteristics of the resonator were investigated as follows. The resonator is driven with a T3 electrode and the amplitude of the vibration is detected with a T1 electrode. Applied amplitude voltage was varied to 5, 3, and $1 \mathrm{~V}$. The offset voltage is fixed to $5 \mathrm{~V}$. Driving frequency was swept up from $1 \mathrm{kHz}$ to $5 \mathrm{kHz}$. As shown in Fig.5, three resonant peaks were observed. A first peak at $3.42 \mathrm{kHz}$

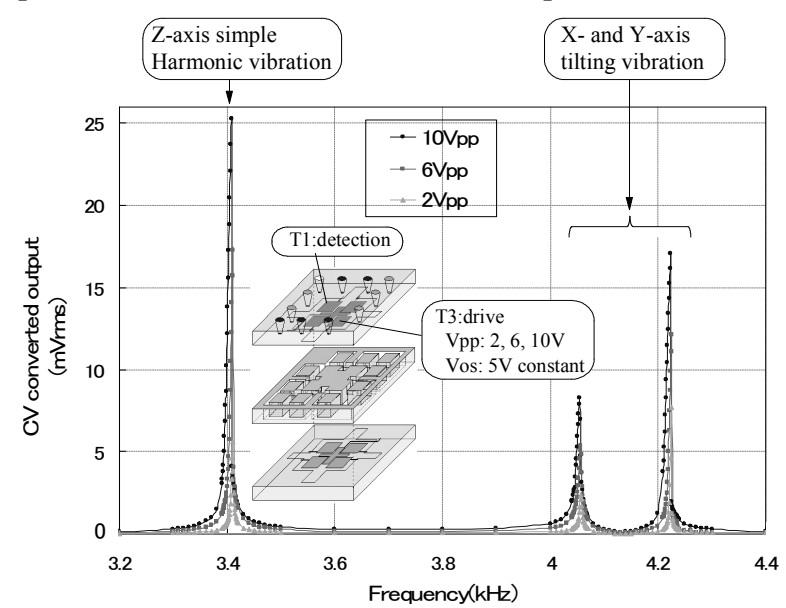

Fig.5 Vibration characterisitics of the resonator

corresponded to the resonance of $\mathrm{Z}$-axis simple harmonic vibration, and the others of $4.05 \mathrm{kHz}$ and $4.22 \mathrm{kHz}$ corresponded to the resonance of the two tilting vibration around $\mathrm{X}$-and $\mathrm{Y}$-axis. This split into two peaks is considered to be caused by imbalance of the four silicon beams. It is quite difficult to merge the resonant peaks of tilting vibration around $\mathrm{X}$-and $\mathrm{Y}$-axis into a peak completely. Although it is disadvantageous for high sensitive detection, the non-resonant detection mode has an advantage of low mechanical coupling. It is also favorable that extreme high precise fabrication process is not necessary. In addition, the peak height decreases as increasing inner pressure generally [5]. However, these peak profiles have not changed for a year, therefore the sensor is considered to be sealed without leakage.

\section{3-axis acceleration characteristics}

The static acceleration characteristics in the cases of electrostatic drive and non-drive conditions were investigated by rotating along each axis under gravitational $1 \mathrm{G}$. The electrostatic reference vibration was carried out by applying $5 \mathrm{Vpp}+5 \mathrm{Vos}$ to $\mathrm{T} 0$ and $\mathrm{B} 0$ electrodes. The driving frequency was approximately $3.42 \mathrm{kH}$ which was $\mathrm{Z}$-axis resonant frequency. Measured acceleration characteristics are shown in Fig.6. Each output voltage increase in proportion to applied acceleration. Each axis acceleration sensitivities under electrostatic drive tends to be several \% higher than that under non-drive. It seems that the averaged capacitance under electrostatic drive becomes lager than that under non-drive because the capacitance gap is changing according to amplitude of the vibration. The $\mathrm{X}$-axis

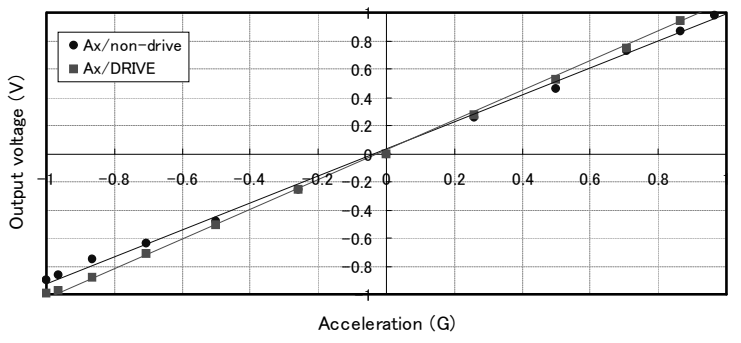

(a) X-axis acceleration

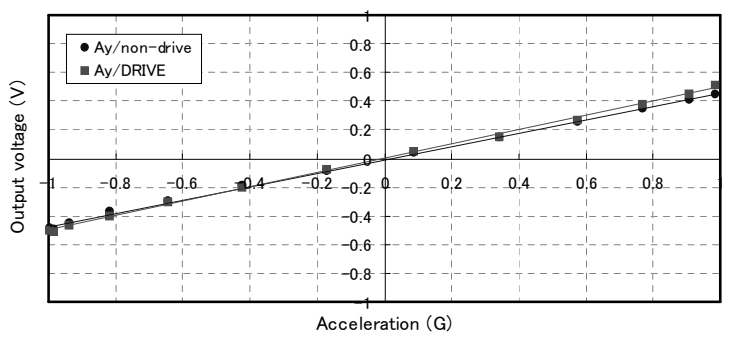

(b) Y-axis acceleration

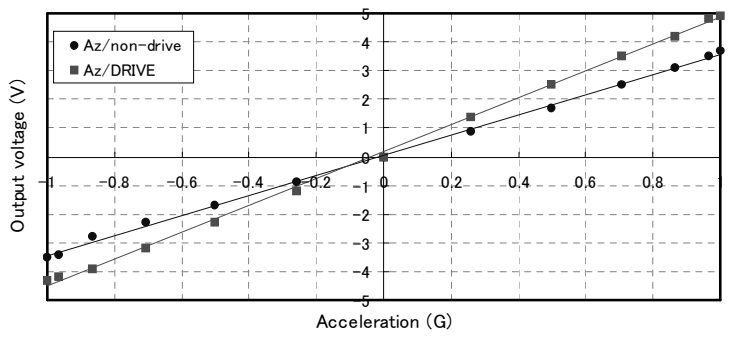

(c) Z-axis acceleration

Fig.6 3-axis acceleration characteristics 
sensitivity is higher than that of $\mathrm{Y}$-axis, because the initial gap used for the $\mathrm{X}$-axis detection is narrower than that of $\mathrm{Y}$-axis. Cross axis sensitivity was less than $5 \%$ and non-linearity was less than $2 \%$ F.S. Measured 3-axis acceleration characteristics are summarized in Table 1.

Table $1 \quad 3$-axis acceleration characteristics

\begin{tabular}{ccc}
\hline Sensitivity $(V / G)$ & non-drive & DRIVE \\
\hline Ax & 1.86 & 1.93 \\
Ay & 0.95 & 1.06 \\
Az & 3.51 & 4.67 \\
\hline Non-linearity & \multicolumn{2}{c}{$<2 \%$ f.s. } \\
\hline Cross-axis sensitivity & \multicolumn{2}{c}{$<5 \%$} \\
\hline
\end{tabular}

\section{2-axis angular rate characteristics}

The angular rate characteristics were measured by making a half rotation of a turn table with a reference gyro sensor setting nearby. The electrostatic resonant drive was carried out under the same condition indicated in a previous section. Measured angular rate signals of the developed sensor and the reference gyro sensor are shown in Fig.7 (a). The signals of the developed motion

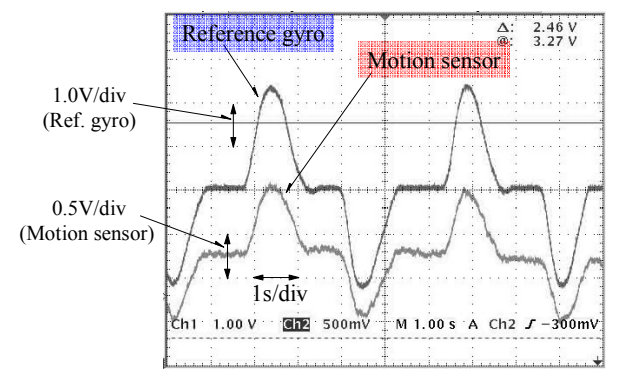

(a) Measured angular rate signal

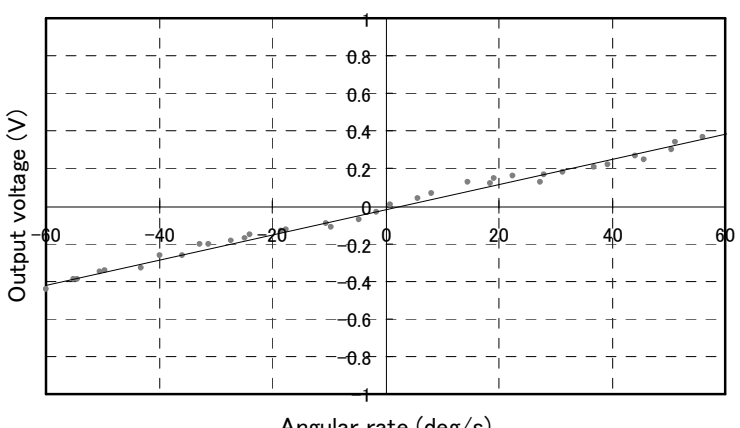

(b) $\mathrm{X}$-axis angular rate

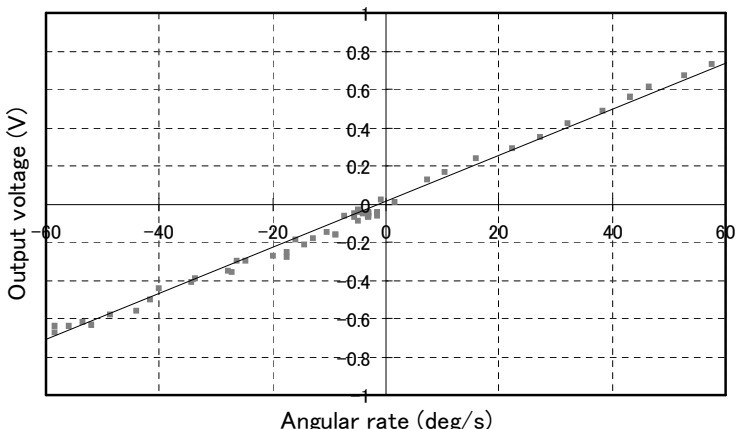

(c) Y-axis angular rate

Fig.7 2-axis angular rate characteristics sensor follow that of the reference gyro sensor entirely with low noise. As shown in Fig.7 (b) and Fig.7 (c), each axis output voltage increase in proportion to applied angular rate. Measured 2-axis angular rate characteristics are summarized in Table 2. In spite of the non-resonant detection mode, stable detection of over $6 \mathrm{mV} /[\mathrm{deg} / \mathrm{s}]$ can be obtained. The $\mathrm{X}$-axis sensitivity is lower than that of $\mathrm{Y}$-axis because of the similar reason to acceleration detection. The same as acceleration detection, 2-axis sensitivities can be equalized by optimization of the initial gap of top side and bottom side. Cross axis sensitivity was less than $5 \%$ and non-linearity was less than $3 \%$ F.S.

Table 2 2-axis angular rate characteristics

\begin{tabular}{cc}
\hline Sensitivity $(\mathrm{mV} /[\mathrm{deg} / \mathrm{s}])$ & \\
\hline$\Omega_{\mathrm{x}}$ & 6.67 \\
$\Omega_{\mathrm{y}}$ & 12.1 \\
\hline Non-linearity & $<3 \%$ f.s. \\
\hline Cross-axis sensitivity & $<5 \%$ \\
\hline
\end{tabular}

\section{CONCLUSIONS}

We have developed a new 5-axis motion sensor fabricated by SOI bulk-micromachining using deep RIE and vacuum anodic bonding processes. By using electrostatic resonant drive at $3.42 \mathrm{kHz}$ and non-resonant capacitive detection mode, we have achieved stable detection of 3-axis acceleration and 2-axis angular rate. Measured sensitivity of the 3 -axis acceleration and the $2-$ axis angular rate are over $1 \mathrm{~V} / \mathrm{G}$ and over $6 \mathrm{mV} /[\mathrm{deg} / \mathrm{s}]$, respectively.

\section{REFERENCES}

[1] N.Taniguchi, H.Itano, K.Okada : "The 5-Axis Motion Sensor" Technical Digest of the 16th Sensor Symposium, pp.41-44, 1998.

[2] Y.Watanabe, T.Mitsui, T.Mineta, S.Kobayashi, N.Taniguchi, K.Okada : "Five-Axis Capacitive Motion Sensor with Electrostatic Drive and Capacitive Detection Fabricated by Silicon Bulk Micromachining" , Sensors and Actuators A97-98, pp.109-115, April 2002.

[3]K.Maenaka, T.Fujita and M.Maeda : "Design Problems on Vibratory Micro-Gyroscopes" , T.IEE Japan, Vol.118-E, No.7/8, pp.377-383, 1998.

[4] K.Ikeda and K.Maenaka : "Sensor Technologies Based on Mechanical Resonance and Vibration" , T.IEE Japan, Vol.120-E, No.3, pp.87-92, 2000.

[5] T.Mineta, S.Kobayashi, Y.Watanabe, S.Kanauchi, I.Nakagawa, E.Suganuma and M.Esashi : "Three-Axis Capacitive Accelerometer with Uniform Axial Sensitivities " Journal of Micromechanics and Microengineering, pp.431-435, Vol.6, 1996. 